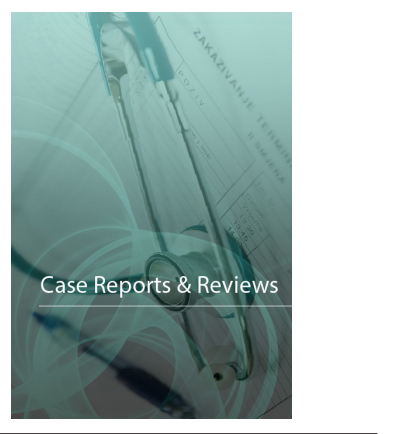

Correspondence

Dr. Gianluca Sottilotta

Viale Europa 89127 Reggio Calabria, Italy

Tel: +390965393841

Fax: +390965393852

E-mail: gianluca.sottilotta@virgilio.it

- Received Date: 25 Nov 2020

- Accepted Date: 08 Dec 2020

- Publication Date: 12 Dec 2020

Copyright

(c) 2020 Science Excel. This is an openaccess article distributed under the terms of the Creative Commons Attribution 4.0 International license.

\title{
Management of heparin in COVID-19 patients during the first phase of pandemic
}

\author{
Gianluca Sottilotta' , Carmelo Mangano² ${ }^{2}$ Rosa Basile², Francesca Luise ${ }^{3}$, \\ Demetrio Megalizzi ${ }^{1}$, Giovanna Maria Nicolò ${ }^{4}$, Vincenzo Orianaa ${ }^{1}$, Angela \\ Piromalli ${ }^{3}$
}

${ }^{1}$ Hemophilia Centre, Thrombosis and Haemostasis Service, Grande Ospedale Metropolitano, Reggio Calabria, Italy ${ }^{2}$ Unit of Infectious Disease, Grande Ospedale Metropolitano, Reggio Calabria, Italy

${ }^{3}$ Analysis Laboratory, Grande Ospedale Metropolitano, Reggio Calabria, Italy

${ }^{4}$ Clinical Pathology and Clinical Biochemistry, University of Catania, Italy

\begin{abstract}
The coronavirus disease (COVID-19) is associated with a significant risk of thrombotic complications. We reported our first wave experience regarding the use of Low Molecular Weight Heparin (LMWH) and Fondaparinux in Covid-19 patients admitted to our Hospital. The aim of our study was to evaluate the effectiveness and safety of increased LWMH prophylaxis. 57 patients admitted to our hospital for COVID-19, median age 65, were studied from March to May 2020. We observed one thrombotic event and 3 bleedings. The incidence of thrombosis was 1.7\%: this result is much lower than that reported by other studies regarding patients untreated with heparin or in prophylaxis at standard dosages. LMWH therapy has proven effective and safe in terms of thrombosis prevention and has contributed to the favourable outcome of hospitalizations.
\end{abstract}

\section{Introduction}

Recent clinical data has highlighted that coronavirus disease 2019 (COVID-19) is associated with a significant risk of thrombotic complications ranging from microvascular thrombosis, venous thromboembolic disease, and stroke [1-3]. Several studies have reported a cumulative incidence of VTE between 25\% and $49 \%$ in patients with severe COVID-19, and pulmonary embolism was evidenced as the most common thrombotic complication [46]. Various hypotheses have been considered to explain the correlation between COVID-19, hypercoagulability and high risk of thrombosis. The clinical evolution of COVID-19 infection seems to be framed in 3 phases, one of which is initial, characterized by the intracellular replication of the virus, with symptoms such as general malaise, fever and dry cough; a second phase with alterationsinlung function determined both by the pathological action of the virus and by the immune response of the subject affected by COVID-19, and which is characterized by an almost always bilateral interstitial pneumonia picture; this, if initially manifested without hypoxemia, can evolve towards a worsening clinical picture dominated by the cytokine storm and the consequent hyperinflammatory state: this can cause in the lungs, pictures of arterial and venous vasculopathy with thrombus of small vessels. The final stages of this very serious clinical picture lead to severe acute respiratory distress syndrome and in some cases to disseminated intravascular coagulation. In the clinically more advanced stages, in addition to the progressive alteration of the inflammatory indices, a worsening of haemocoagulation parameters, expression of excessive activation of coagulation and in some cases of consumption of factors was also highlighted. These observations were made for the first time during the SARS epidemic in 2002-2003, a time when the first reports on the efficacy of heparin treatment in this type of patient date back. Increased VTE events in COVID-19 are thought to be due to immobilization, excessive inflammation, and diffuse intravascular coagulation (DIC). Although not primarily a thrombotic process, inflammation and hypoxia with acute lung injury leads to a profound inflammatory state due to cytokine storm, macrophage, and endothelial activationrelated processes associated with a surge in IL-1, IL-6, IL-8, and TNF-alpha which suggest that there are biological evidences for the thrombotic process. Recent studies hypothesized that the IL- 6 excess stimulates megakaryocytopoiesis and platelets production in the bone marrow and particularly in the lungs, the target organs of SARS-CoV-2 infection at the beginning; the subsequent in loco release of activated platelets may induce an hypercoagulability state prone to abnormal immunothrombosis with blood oxygenation worsening and hypoxic tissue injury [7,8]. Evidence of coagulopathy has been reported, with patients demonstrating often markedly elevated serum levels of D-dimer, lactate dehydrogenase, and total bilirubin with slight prolongation or no changes in partial thromboplastin time (PT) or activated partial thromboplastin time (PTT)[9]. Diffuse microvascular thrombi with possible thrombotic microangiopathy in multiple organs have been reported on autopsy review without viral infiltrates. For all these reasons, the use of heparin (preferably the low molecular weight one) for the prevention 
of thromboembolism is currently recommended in COVID-19 patients. In fact, in addition to the function of anticoagulant, heparin would also have an anti-inflammatory action through various mechanisms such as: inhibition of thrombin synthesis, binding with inflammatory cytokines, inhibition of neutrophilic chemotaxis and leukocyte migration and neutralization of C5a peptide. It also seems, from studies conducted on animal models, that heparin may also have an antiviral role: it is known, in fact, that its molecules would bind to circulating coronaviruses, thus preventing their binding to the heparan-sulphate proteoglycans present on the surface of cells, and thus effectively preventing the penetration of the virus inside the cell[10]. Emerging data suggests that deregulated thrombin generation and abnormal activation of the coagulation cascade can lead to the development of disseminated intravascular coagulation (DIC) and is associated with worsening pneumonia and mortality [11]. We reported our first wave experience regarding the use of Low Molecular Weight Heparin (LMWH) and Fondaparinux in COVID-19 patients admitted to our Hospital. The aim of our study was to evaluate the effectiveness and safety of increased LWMH prophylaxis: this was performed by estimating the incidence of thrombotic and haemorrhagic events in hospitalized patients, by evaluating if the presence of thrombocytopenia virus-related may increase the risk of bleeding and by investigating if higher LWMH dosages could be correlated with shorter hospitalization times.

\section{Methods}

57 patients (36 M, $21 \mathrm{~F}$ ) for COVID-19, median age 65 (19-92) were studied from March to May 2020. The average hospitalization was 27 days (5-64). All patients underwent LWMH prophylaxis from the first day of admission, according to the local guidelines. The LWMH dosage ranged between normal, increased prophylactic or therapeutic doses depending on patients' weight and the presence of one or more cardiovascular or thromboembolic risk factors. The used treatment was enoxaparin $(51 / 57$ patients, $89.5 \%)$, nadroparin $(2 / 57$, $3.5 \%)$, and fondaparinux $(4 / 57,7 \%)$. Of the 51 enoxaparin patients, 35 received 4000 IU bid, 2 received 5000 IU bid, 10 were treated with $6000 \mathrm{IU}$ bid, 1 with $8000 \mathrm{IU}$ bid, 3 patients received $4000 \mathrm{IU}$ once a day. Fondaparinux was used in 3 patients at a dosage of $2.5 \mathrm{mg}$ and in one at a dosage of $1.5 \mathrm{mg}$. Nadroparin was only administered a single daily dose of $2850 \mathrm{IU}$ and $5700 \mathrm{IU}$.

\section{Results}

Of the 57 studied patients, 2 died for disseminated Intravascular coagulation (DIC), the other 55 have been discharged. We observed one thrombotic event (cerebral ischemia) and 3 bleedings: a patient with iliopsoas muscle hematoma, and another with both haematuria and iliopsoas hematoma. Ten episodes of thrombocytopenia, not LWMH-related, were observed: all of them were mild or moderate, except one severe thrombocytopenic myelodysplastic patient: in only one case was a moderate thrombocytopenia associated with bleeding. In $7 / 10$ cases the thrombocytopenia was transient. We did not find any correlation between increased antithrombotic prophylaxis and hospitalization reduction: patients on enoxaparin 4000 bid had an average hospital stay of 25 days, fewer than patients on 6000 IU bid (32 days).

\section{Conclusions}

The incidence of thrombosis was 1.7\%: this result is much lower than that reported by other studies regarding patients untreated with heparin or in prophylaxis at standard dosages. The three bleedings occurred in 2/57 (3.4\%) patients, both treated with enoxaparin 6000 IU bid. LMWH therapy has proven effective and safe in terms of thrombosis prevention and has contributed to the favourable outcome of hospitalizations, according to what is currently known about the COVID-19 etiopathogenesis and symptoms. The low incidence of bleeding could be further reduced by monitoring the platelet count for early reducing the LWMH dosage in case of thrombocytopenia and, if possible, also evaluating the Anti-Xa activity.

\section{Conflict of Interest}

The authors declare no conflict of interest. All the authors were responsible for data collection and contributed to the drafting and final revisions of the manuscript.

\section{References}

1. Rali P, O'Corragain O, Oresanya L, et al. Incidence of Venous Thromboembolism (VTE) in COVID-19: An experience from a single large academic center. J Vasc Surg Venous Lymphat Disord. 2020;S2213-333X(20)30524-2.

2. Longhitano $\mathrm{Y}$, Racca F, Zanza C, et al. Venous thromboembolism in critically ill patients affected by ARDS related to COVID-19 in northern-west Italy. Eur Rev Med Pharmacol Sci. 2020;24(17):9154-9160.

3. McFadyen JD, Stevens H, Peter K. The emerging threat of (micro) thrombosis in COVID-19 and its therapeutic implications. Circ Res. 2020;127(4):571-587.

4. Cui S, Chen S, Li X, Liu S, Wang F. Prevalence of venous thromboembolism in patients with severe novel coronavirus pneumonia. J Thromb Haemost. 2020;18:1421-1424.

5. Klok FA, Kruip MJHA, van der Meer NJM, et al. Incidence of thrombotic complications in critically ill ICU patients with COVID- 19. Thromb Res. 2020;191:145-147.

6. Klok FA, Kruip MJHA, van der Meer NJM, et al. Confirmation of the high cumulative incidence of thrombotic complications in critically ill ICU patients with COVID-19: an updated analysis. Thromb Res. 2020;191:148-150.

7. Roncati L, Ligabue G, Nasillo V, et al. A proof of evidence supporting abnormal immunothrombosis in severe COVID-19: naked megakaryocyte nuclei increase in the bone marrow and lungs of critically ill patients. Platelets. 2020;31(8):1085-1089.

8. Roncati L, Manenti A, Manco G, Farinetti A. Abdominal aortic thrombosis complicating COVID-19 pneumonia. Ann Vasc Surg. 2020;67:8-9.

9. Shi $\mathrm{C}$, Wang $\mathrm{C}$, Wang $\mathrm{H}$, et al. The potential of low molecular weight heparin to mitigate cytokine storm in severe COVID-19 patients: A retrospective cohort study. Clin Transl Sci. 2020;13(6):1087-1095.

10. Milewska A, Zarebski M, Nowak P, Stozek K, Potempa J, Pyrc K. Human coronavirus NL63 utilizes heparan sulfate proteoglycans for attachment to target cells. J Virol. 2014;88(22):13221-30.

11. AlSamman M, Caggiula A, Ganguli S, Misak M, Pourmand A. Non-respiratory presentations of COVID-19, a clinical review. Am J Emerg Med. 2020;S0735-6757(20)30847-0. 